\title{
PRELIMINARY EVALUATION OF LANDSAT 4 \& 5 DATA OF ANTARCTICA
}

\section{(Abstract)}

by

Jane G. Ferrigno

(U.S. Geological Survey, Reston, Virginia 22092, U.S.A.)

\section{ABSTRACT}

Landsats 4 \& 5 have acquired approximately one thousand images of Antarctica in 1984 and 1985, including a few thematic mapper images. I will show, on two maps, where these images have been acquired and give a preliminary evaluation of their quality.

\section{MASS BALANCE ALONG TWO TRANSECTS OF THE GREENLAND ICE SHEET}

\author{
(Abstract) \\ by \\ J.M. Kostecka and I.M. Whillans
}

(Department of Geology and Mineralogy and Institute of Polar Studies, The Ohio State University, 125 South Oval Mall, Columbus, OH 43210, U.S.A.)

\section{ABSTRACT}

The mass balance and sensitivity of calculated mass balance to uncertainties in the data and in the model for the variation of ice velocity with depth are addressed, using data from the EGIG transect and from the OSU transect in south Greenland (Drew and Whillans, 1984). The calculation uses a non-steady continuity model with allowances for three-dimensional flow and horizontal velocity variation with depth. Depth variation in horizontal velocity is obtained, using the constitutive relation for ice with calculated temperature profiles and with full allowance for longitudinal stresses and enhancement of flow due to ice anistrophy and texture. Separate calculations are made for different thickening or thinning rates, until a match between observed and calculated surface velocities is obtained.

For the EGIG transect, our mass-balance results are in the range reported by Mälzer and Seckel (1975) and Bindschadler (1984). Results for the OSU transect, just south of the Arctic Circle on the south dome, are also reported.

Of particular interest is the sensitivity study, which is designed to determine which aspects of the data and flow behavior are most critical to the calculations. Data that are comparatively well-constrained are flow-line definition, surface velocity and thickness. The flow lines and lateral spreading are obtained from satellite radar altimetry (Zwally and others, 1983) and checked against velocity data along the OSU profile. Thicknesses are from aerial radar sounding (Overgaard, personal communication). Surface velocities along the EGIG transect are corrected in a manner similar to that suggested by Robin (1983, figure 2.17b) and possible errors are not critical. Along the OSU transect the surface velocities are well-determined by Doppler satellite tracking in short-arc translocation mode.

Accumulation rates are not well-determined and show substantial scatter for reasons that are not understood. Along the OSU transect, accumulation rates were obtained by augering for the nuclear bomb horizons. As reported earlier (Mock, 1967), the accumulation rates in this region show no clear pattern with elevation or slope. Similar, but less severe, problems exist in the EGIG area. The lack of a good model describing the geographic variation in accumulation rate results in calculated surface velocity uncertainties of $9 \%$ for the OSU and $6 \%$ for the EGIG transect.

The calculated results are comparatively insensitive to parameters affecting the temperature profile. This is because, in this model, the mean velocity is determined by continuity and temperature affects only the ratio of horizontal velocity at a given depth to the mean velocity for the entire profile.

Longitudinal stresses are included because they affect the viscosity through the effective shear stress. Neglecting this would lead to a $30 \%$ error in calculated surface velocity near the ice divide and about $6 \%$ away from the divide. Provided longitudinal stresses are included, uncertainties in these stresses are not critical.

Enhancement factors are, however, important. Most authors believe that ice fabric, texture, and impurity content can affect strain rates by $100 \%$ to $1200 \%$ (Shoji and Langway, 1984). As with the temperature profile, this problem does not affect calculated mean velocities, which are based on continuity, but it does affect the ratio of surface to mean velocity. Enhancement factors are more critical than temperature uncertainties and therefore failure to include depth-variable flow enhancement alters calculated surface velocities by 5 to $10 \%$.

In summary, the mass balance along two transects has been investigated by comparing measured and calculated surface velocities. The most critical problems are (1) understanding the geographic distribution in accumulation rate and (2) correctly allowing for enhanced shearing due to structural variations with depth. 